\title{
Incidencia de los distintos tipos de violencia escolar en Educación Primaria y Secundaria
}

\section{Víctor Domínguez Rodríguez}

Universidad de Vigo, España

mail: jdalonsodedu.xunta.es

ORCID: https://orcid.org/0000-0002-2521-0923

Manuel Deaño Deaño

Universidad de Vigo, España

mail: deanoduvigo.es

ORCID: https://orcid.org/0000-0003-4451-0275

\author{
Fernando Tellado González \\ Universidad de Vigo, España \\ mail: ftelladoduvigo.es \\ ORCID: https://orcid.org/0000-0003-1912-9018
}

\section{RESUMEN}

El objetivo del estudio era cuantificar los diferentes tipos de violencia escolar que se llevan a cabo en centros de primaria y secundaria, así como analizar si las variables socio-escolares determinan y predicen su desarrollo en estudiantes. Se llevó a cabo una investigación no experimental, descriptiva, inferencial y de regresión. La muestra, intencional y estratificada, fue de 1139 participantes [423 alumnos de primaria $(M=10.56 ; D T=.67)$ y 716 alumnos de secundaria $(M=14.09 ; D T=1.22)]$, a los cuales se administra un cuestionario de violencia escolar CUVE-R. Los resultados evidenciaron que ambas etapas educativas señalan una mayor presencia de violencia verbal, violencia que ejerce el profesorado y violencia física, sin embargo, dichas conductas violentas eran más frecuentes en la etapa de educación secundaria obligatoria. Asimismo, se constatan diferencias significativas en sexo (mayor presencia de violencia del profesorado o exclusión social en los hombres, y violencia verbal o disrupción en las mujeres), edad (a mayor edad se incrementan los tipos de violencia escolar), etapa educativa (mayor aparición de violencia escolar en la etapa de educación secundaria obligatoria) y expediente académico (se incrementa la violencia escolar en el alumnado cuando son bajos expedientes académicos). Finalmente, la violencia física y verbal son las variables de mayor potencialidad predictiva en la violencia escolar. En conclusión, los datos sugieren la necesidad de identificar y atender los diferentes tipos de violencia escolar, así como tener en cuenta las variables socio-escolares que puedan estar mediando en este problema, para una intervención posterior a nivel psicosocioeducativo.

Palabras clave: violencia escolar, educación primaria, educación secundaria obligatoria (ESO), centros educativos, metodología cuantitativa.

\section{Incidence of different types of school violence in primary and secondary education}

\begin{abstract}
The objective of the study is to quantify the different types of school violence that take place in primary and secondary schools, as well as to analyze whether the socio-school variables determine and predict their development in students. A non-experimental, descriptive, inferential and regression investigation was carried out. The intentional and stratified sample comprised 1139 participants [423 primary education students $(\mathrm{M}=10.56$; $\mathrm{DT}=.67)$ and 716 secondary education students $(\mathrm{M}=14.09 ; \mathrm{DT}=1.22)$ ], to which is administered the questionnaire of school violence (CUVE-R). Results show that both educational stages indicate a greater presence of verbal violence, violence by teachers and physical violence, however, these violent behaviors are more frequent in the compulsory secondary education stage. Likewise, significant differences are found in sex (greater presence of violence by teachers or social exclusion in men, and verbal violence or disruption in women), age (the older the types of school violence increase), educational stage (greater appearance of school violence in the compulsory secondary education stage) and academic record (school violence increases in students with low academic records). Finally, physical and verbal violence are the variables with the greatest predictive potential in school violence. In conclusion, data suggest the need to identify and address the different types of school violence, as well as to take into account the socio-school variables that may be mediating this problem, for a subsequent intervention at the psychosocial and educational level.
\end{abstract}

Key words: school violence, primary education, compulsory secondary education (CSE), educational centers, quantitative methodology. 


\section{Introducción}

Los centros educativos consuman un rol prioritario en la socialización del individuo y se constituyen en el espacio donde niños / as y adolescentes pasan gran parte de su tiempo avanzando en sus habilidades cognitivas y no cognitivas para el desarrollo en todas las etapas evolutivas. En dichos centros de enseñanza, empieza el primer acercamiento al mundo externo (niñez) y ocurre la transición a la madurez completa (adolescencia). No obstante, en las comunidades educativas, al igual que en la sociedad, surgen conflictos (entendidos como "combate, lucha, pelea o situación de difícil salida -RAE, 2019-), que mal gestionados pueden derivar en problemas de convivencia, dando lugar al fenómeno de la violencia escolar (Albaladejo-Blázquez, Ferrer-Cascales, Reig-Ferrer y Fernández-Pascual, 2013).

Desde hace años, la violencia se ha ido conformando como uno de los puntos cruciales y más sensibles en el campo educativo. La Organización de las Naciones Unidas para la Educación, Ciencia y Cultura (UNESCO, 2019) sostiene que la violencia escolar en todas sus formas constituye una violación de los derechos en la infancia o adolescencia a la educación, salud y bienestar. No obstante, aunque se constate que la violencia entre escolares es un hecho habitual en los espacios de enseñanza (Jiménez y Lehalle, 2012; Juvonen y Graham, 2014; Martínez-Baena y Faus-Boscá, 2018), se pueden observar visiones diferenciadas sobre su incidencia. Por un lado, estudios que apuntan bajos niveles de violencia escolar en educación primaria y secundaria que no tienen por qué entorpecer la convivencia en los centros educativos (Ceballos, Cerezo, 2009). Por otro, trabajos que muestran su incremento en los jóvenes llegando a ser considerada como un problema sistémico que afecta a la labor educativa (Álvarez-García, Dobarro, Álvarez, Núñez y Rodríguez, 2014; Merma, Peiró y Gavilán, 2013). También destacan las investigaciones que han indicado problemas de comportamiento a edades tempranas, acentuándose en el periodo adolescente de manera más grave (Kim, Gilman, Hill y Hawkins, 2016; Loeber, Capaldi y Costello, 2013). Finalmente, no faltan estudios que han señalado una mayor incidencia de la violencia escolar según la etapa educativa, unos indicando la etapa de primaria como la de mayor presencia de acoso escolar (Pérez, 2015; Weimer y Moreira, 2014) y otros apuntando como la más propensa la educación secundaria obligatoria (Giménez, Arnaiz, Cerezo y Prodocimo, 2018; Sánchez, Vallejo y Roig, 2019).

En este contexto, la violencia puede ser entendida como "cualquier acción que ocurra y se geste en la escuela, dificultando su finalidad educativa y lesionando la integridad de algún miembro de la comunidad escolar" (Pacheco-Salazar, 2018, p. 113). Por consiguiente, para poder considerar un comportamiento como violencia escolar, debe ser una acción dañina e intencional a manos de una o más personas dirigido contra otra que tiene dificultad para defenderse (Olweus 2006). Así pues, las conductas violentas pueden presentarse a través de distintas formas o maneras (Salazar y González, 2019), las cuales han progresado desde las agresiones físicas, verbales, o psicológicas, hacia maneras que actualmente se encuentran en pleno auge como las ejercidas mediante el uso del teléfono móvil, tablet u otros dispositivos portátiles conectados a internet (Rivera, Reynoso y Vilchis, 2018).

Teniendo en cuenta a Cobo y Tello (2011), para que un fenómeno se considere violencia escolar debe tener las siguientes condiciones: ser una acción agresiva e intencionalmente dañina, molesta a otro sin que haya provocación, se presente de forma repetida, hay un desequilibrio de poder y provoca un daño emocional. En este escenario, se desarrollan diferentes teorías expli- cativas que fundamentan la violencia escolar. Por un lado, las teorías innatas que consideran la agresividad como parte orgánica del individuo o sus impulsos internos (genética, etológica, psicoanalítica, señal de activación). Por otro, las teorías ambientales que entienden el medio ambiente que rodea a las personas como el origen de la agresividad (aprendizaje social, sociológica, ecológica).

En este estudio, se va analizar la violencia escolar desde un modelo ecológico (Teoría ecológica de Bronferbrenner) al considerar que es necesario estudiar las relaciones de los estudiantes con el ambiente que les rodea incluyendo las interconexiones e influencias de las mismas. Es decir, la violencia escolar es entendida desde el contexto sociescolar en el cual tiene lugar (Debarbieux y Blaya, 2010). En consecuencia, resulta evidente que la determinación de los tipos de violencia escolar está en función del concepto de violencia y del papel que ésta va a desempeñar en los centros de enseñanza.

Así pues, son numerosos los estudios que hacen referencia a la incidencia de diferentes tipos de violencia escolar y analizan su relación con diversas variables socio-demográficas. Por un lado, aparecen trabajos que consideran que la prevalencia del tipo de violencia escolar está influenciada por la población del contexto en el cual se observa. Por otro, los que sostienen que factores como el sistema económico-social, menoscabo del respeto y valores básicos de convivencia, mayor complejidad y heterogeneidad social, pérdida de liderazgo educativo en los sistemas tradicionales de educación -familia y sistema educativo-, o una mayor aparición y visibilidad de la violencia, tienen influencia en el sistema educativo y conforman en mayor o menor medida la convivencia de los mismos (Jares, 2006)

El Instituto Vasco de Evaluación e Investigación Educativa en su informe sobre "el maltrato entre iguales en educación primaria y educación secundaria obligatoria" (2012), señala que las conductas de mayor incidencia son las de maltrato verbal como los insultos, poner motes o hablar mal. En esta misma línea, se encuentra el trabajo realizado por Álvarez-García, Álvarez, Núñez, González-Pienda, González-Castro y Rodríguez (2008) al indicar que el tipo de violencia más habitual por parte del alumnado es la verbal. No obstante, el estudio en centros de educación especial de Falla, Alós, Moriana y Ortega (2012) señala la prevalencia de cometer o padecer violencia directa, tanto física como verbal. Del mismo modo, son numerosas las investigaciones que sostienen una mayor prevalencia de la violencia verbal y física en los centros de enseñanza (Ayala-Carrillo, 2015; Calderón, Vera y Llano, 2018; Conde y Ávila, 2019; Kuppens, Grietens, Onghena, Michiels y Subramanian, 2008; Sapién-Zúñiga, Ledezma-Rivas y Ramos-Trevizo, 2019)

Asimismo, ya es una realidad y continúa en aumento paulatino la violencia ejercida a través de las Tecnologías de la Información y la Comunicación (Rivera, Reynoso y Vilchis, 2018; Cerezo-Ramírez, 2017). De hecho, Calmaestra, Escorial, García, Del Moral, Perazzo y Ubrich (2016), o Liébana, Deu del Olmo y Real (2015) señalan que el tipo de violencia que se da en mayor medida se corresponde con la violencia a través de las TIC. Sin embargo, las investigaciones realizadas por Gutiérrez (2019) o Garaigordobil, Martínez y Machimbarrena (2017) encuentran que el tipo de violencia con mayor presencia es la violencia del profesorado hacia el alumnado. A su vez, López (2012) determinó que la causa más frecuente de violencia en los centros educativos era el uso de apodos entre estudiantes, mientras que Coarite y Carrillo (2014) concluyen que se producen en mayor medida cuando los propósitos, intereses o información que manifiestan los jóvenes son interpretados como provocaciones por otras personas. 
Por otra parte, determinados estudios ponen de manifiesto las variables socio-demográficas que pueden estar asociadas con los tipos de violencia escolar. Así pues, León-Moreno, Martínez-Ferrer, Musitu-Ochoa y Moreno-Ruiz (2019) indican diferencias estadísticamente significativas entre chicos y chicas en la relación entre victimización y benevolencia. También, Medina y Reverte (2019) señala que los varones muestran mayores niveles de victimización, especialmente en violencia física y violencia a través de las TIC, siendo en secundaria donde las mujeres presentan tasas más bajas de victimización escolar. Además, García-Fernández, Romera-Felix y Ortega-Ruiz (2017) han hallado en una muestra de 1278 alumnos/as (10-14 años) una mayor cibervictimización en hombres. En este sentido, Domínguez-Alonso, López-Castedo y Nieto-Campos (2019) en una muestra de 4467 alumnos/as (12-16 años) evidencian una ligera prevalencia del género femenino sobre el masculino en las acciones que conforman los diferentes tipos de violencia, los chicos están más implicados en la violencia ejercida por el profesorado, violencia física y exclusión social, mientras que las chicas lo hacen en violencia verbal, violencia del alumnado hacia el profesorado y disrupción en el aula. La UNESCO (2019) indica que el acoso físico es más común entre los niños, mientras que el psicológico es más frecuente entre las niñas.

Si se hace referencia a la etapa educativa, estudios como los realizados por Weimer y Moreira (2014) o Pérez (2015), afirman que es la etapa de Educación Primaria Obligatoria (6-12 años) donde los casos de acoso escolar muestran su mayor presencia. Asimismo, el trabajo realizado por Medina y Reverte (2019) sobre la incidencia de victimización en primaria y secundaria obligatoria indica una prevalencia significativa de victimización individual en la etapa de primaria, con una victimización grupal inter-etapas. No obstante, investigaciones como las realizadas por Giménez et al. (2018) o Sánchez et al. (2019) señalan que es la etapa de Educación Secundaria Obligatoria donde existe una mayor incidencia de violencia escolar.

Finalmente, conviene resaltar que para este estudio se van a tener en cuenta las principales dimensiones de la violencia escolar señaladas por Âlvarez-García, Núñez y Dobarro (2013). Así pues, se evaluará la violencia ejercida por el profesorado hacia el alumnado (conductas injustas como recriminaciones exageradas o inapropiadas), la violencia física (contacto material para producir daño sobre la víctima o pertenencias propiedad de la víctima), violencia verbal (daño producido a través de las palabras como pueden ser los insultos, amenazas y rumores), exclusión social (actos de discriminación y rechazo), disrupción en el aula (el alumnado dificulta al profesorado impartir clase y al resto de alumnos seguir la misma) y violencia ejercida a través de las TIC (amenazas e insultos utilizando el teléfono móvil o internet). El conocimiento de estos tipos de violencia escolar es una primera aproximación al problema, por consiguiente, también constituye un medio para proponer una solución ajustada a la realidad de los contextos escolares.

En consecuencia, asumiendo que la violencia escolar es un asunto compartido que no se resuelve únicamente en los centros educativos, aunque es en ellos donde se libra una acción prolongada y profesionalizada, que con distinto grado de intensidad deja sus huellas en unas comunidades educativas garantes de una convivencia pacífica. La presente investigación surge con el propósito de indagar sobre el tipo de comportamiento violento que prevalece en la enseñanza obligatoria desde la perspectiva del alumnado. De manera más específica, el objetivo principal del estudio es cuantificar la prevalencia del tipo de violencia escolar [Violencia ejercida por el profesorado hacia el alumnado (VPA), Violencia física entre el alumnado (VFA), Violencia ver- bal (VVA), Exclusión social (ES), Disrupción en el aula (DA) y Violencia ejercida a través de las tecnologías de la información y comunicación (VTIC)] en los centros educativos según la etapa educativa (primaria y secundaria). Asimismo, se averiguará la existencia de diferencias significativas en los factores que conforman la violencia escolar (VPA, VFA, VVA, ES, DA, VTIC) en función de las variables socio-escolares (sexo, edad, expediente académico y etapa educativa). En último lugar, se analiza la potencialidad predictiva de las variables (VPA, VFA, VVA, ES, DA, VTIC, sexo, edad, expediente académico y etapa educativa) sobre la violencia que se lleva a cabo en los centros educativos.

Así pues, se somete a consideración (hipótesis de investigación) la predicción de los mismos tipos de violencia escolar para las dos etapas educativas $\left(\mathrm{H}_{1}\right)$ y la existencia de diferencias significativas en las variables socio-escolares analizadas $\left(\mathrm{H}_{2}\right)$. Finalmente se considera que la VPA, VFA, VVA, ES, DA, VTIC, sexo, edad, etapa educativa y expediente académico serán variables predictoras de violencia en los centros educativos $\left(\mathrm{H}_{3}\right)$.

\section{Diseño metodológico}

\section{Participantes}

La muestra (Tabla 1), no probabilística por conveniencia, quedó compuesta por 1139 alumnos de educación primaria (EP) y educación secundaria obligatoria (ESO) de edades comprendidas entre los 9 y 17 años $(M=12,78 ; D T=2,01)$, que pertenecían a quince centros educativos de titularidad pública en la Comunidad Autónoma Gallega.

Tabla 1

Distribución de la muestra

\begin{tabular}{|c|c|c|c|}
\hline & Muestra: 1139 alumnos/as & Frecuencia & Porcentaje \\
\hline \multirow{2}{*}{ Sexo } & Mujer & 570 & $50 \%$ \\
\hline & Hombre & 569 & $50 \%$ \\
\hline \multirow{3}{*}{ Edad } & $<12$ años & 472 & $41.4 \%$ \\
\hline & $12-14$ & 418 & $36.7 \%$ \\
\hline & $>14$ años & 249 & $21.9 \%$ \\
\hline \multirow{3}{*}{$\begin{array}{l}\text { Expediente } \\
\text { académico }\end{array}$} & Siempre ha aprobado & 691 & $60.7 \%$ \\
\hline & $\begin{array}{l}\text { Ha suspendido alguna } \\
\text { materia }\end{array}$ & 177 & $15.5 \%$ \\
\hline & Ha repetido algún curso & 271 & $23.8 \%$ \\
\hline \multirow[b]{2}{*}{ Etapa } & Primaria $\left(5^{\circ}\right.$ y $6^{\circ}$ curso $)$ & 423 & $37.1 \%$ \\
\hline & $\begin{array}{l}\text { Secundaria }\left(1^{\circ}, 2^{\circ}, 3^{\circ} \text { y } 4^{\circ}\right. \\
\text { curso })\end{array}$ & 716 & $62.9 \%$ \\
\hline
\end{tabular}

\section{Instrumento}

Para la realización de este estudio se ha utilizado el cuestionario de violencia escolar (CUVE-R) (Álvarez-García, Núñez, Rodríguez, Álvarez y Dobarro, 2011), que permite analizar la frecuencia de aparición de diferentes tipos de violencia escolar, protagonizado por el alumnado o el profesorado en clases. El cuestionario está formado por 31 ítems redactados en forma de afirmaciones, que se valoran con una escala de tipo Likert $(1=$ nunca, $5=$ siempre). Se ha utilizado la versión de seis factores [VPA (Ítems: 5, 9, 12, 15, 18, 23, 27; ej. “El profesorado tiene manía 
a algunos alumnos o alumnas", VFA (Ítems: 2, 16, 19, 21, 25, 26; ej. "Los estudiantes pegan a compañeros o compañeras dentro del recinto escolar", VVA (Ítems: 1, 3, 7, 10, 14, 17; ej. "Los estudiantes hablan mal unos de otros", ES (Ítems: 4, 8, 29; ej. “Algunos estudiantes son discriminados por sus compañeros o compañeras por su nacionalidad", DA (Ítems: 11, 22, 30; ej. "El alumnado dificulta las explicaciones del profesor o de la profesora con su comportamiento durante la clase", VTIC (Ítems: 6, 13, 20, 24, 28, 31; ej. "Los estudiantes publican en Internet fotos o vídeos ofensivos de compañeros o compañeras"]. En este estudio se ha alcanzado una buena fiabilidad para la muestra total (Alpha de Cronbach $=, 93$ ) y una fiabilidad aceptable para cada uno de los factores (Violencia del profesorado: $\alpha=.86$; Violencia física: $\alpha=$ .71; Violencia verbal: $\alpha=.79$; Exclusión social: $\alpha=.69$; Disrupción en el aula: $\alpha=.74$; Violencia a través de las TIC: $\alpha=.86$ )

\section{Procedimiento}

Seleccionados los centros educativos por conveniencia, se contacto con el equipo directivo con el propósito de explicarles la finalidad y alcance de la investigación y proponerles su participación voluntaria. En primer lugar, se pidió permiso a la dirección de los centros y posteriormente se informó y solicitó el consentimiento a los padres para la participación de sus hijos en el estudio. En segundo lugar, el cuestionario se administró de manera colectiva al alumnado de primaria y secundaria en horario regular de clases, contando con la participación del alumnado cuyos padres dieron su consentimiento para colaborar. Se cumplieron los protocolos éticos de investigación con especial énfasis en la confidencialidad. Todo ello bajo las normas éticas de la Declaración de Helsinki (1975).

\section{Análisis de datos}

En primer lugar, se ha comprobado la percepción del alumnado sobre los diferentes tipos de violencia escolar. Para ello, se tuvo en cuenta las respuestas efectuadas a cada ítem a través de un análisis descriptivo (Medias y desviación típica). Seguidamente, se realizó la prueba $t$ para muestras independientes (variables dicotómicas), análisis de varianza (ANOVA de un factor) y análisis post hoc mediante la prueba Tukey (variables politómicas), para averiguar la existencia de diferencias significativas en los factores que conforman la violencia escolar en función de las variables socio-escolares. Aquí, también se ha calculado el tamaño del efecto ( $\mathrm{d}$ de Cohen): valores entre 0,2 y 0,3 indican un efecto pequeño, alrededor de 0,5 un efecto mediano y mayores que 0,8 un efecto alto (Cohen, 1990). Finalmente, con el propósito de determinar el valor predictivo de diferentes variables (VPA, VFA, VVA, ES, DA, VTIC, sexo, edad, expediente académico y etapa educativa), se ha llevado a cabo un análisis de regresión. Todos los análisis han sido realizados con el paquete estadístico SPSS v. 23 (IBM Corp., 2012), considerando que una relación es estadísticamente significativa cuando $p \leq, 05$.

\section{Resultados}

Análisis descriptivo de las subescalas que conforman la violencia escolar (VPA, VFA, VVA, ES, DA, VTIC)

Al hacer un análisis general de los seis factores que conforman la violencia escolar en primaria y secundaria (Figura 1), se constata de mayor a menor presencia la violencia verbal $(M=$ $13,90)$, violencia del profesorado $(M=12,75)$, violencia física $(M$ $=11,85)$, disrupción en el aula $(M=8,80)$, violencia a través de las
TIC $(M=8,2)$ y exclusión social $(M=5,35)$. Atendiendo a la etapa educativa (primaria y secundaria) se observa una mayor media de violencia escolar en el alumnado de secundaria $(M=11,31, D T$ $=4,33)$ frente al de primaria $(M=8,90, D T=3,24)$.

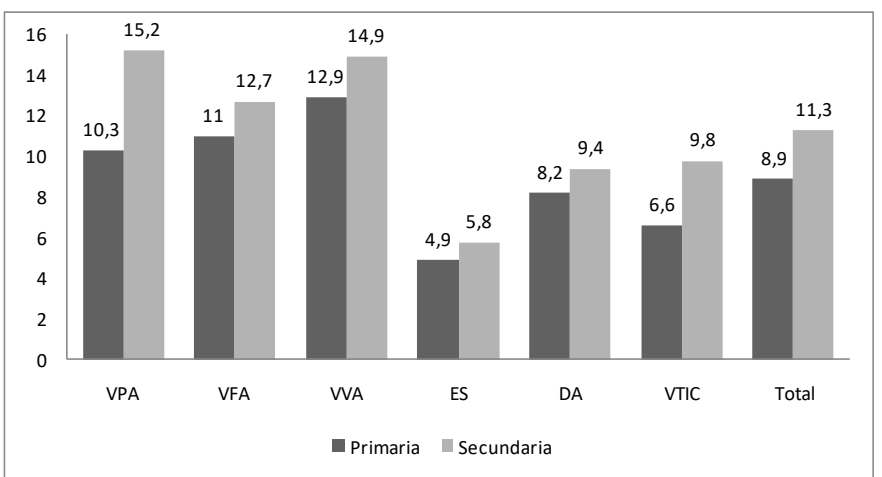

Figura 1. Media de la violencia ejercida por el profesorado hacia el alumnado, violencia física, violencia verbal, exclusión social, disrupción en el aula, violencia a través de las TIC y media total de todos los factores que conforman la violencia escolar en la educación primaria y secundaria obligatoria

Asimismo, presenta una mayor violencia de todos los tipos en la etapa de ESO, siendo más patente en la violencia que ejerce el profesorado sobre el alumnado (diferencia de medias $=4,9$ ) y violencia a través de las tecnologías de la información y comunicación (diferencia de medias $=3,2$ ). Por el contrario, muestra menor diferencia en la exclusión social (diferencia de medias $=, 9$ )

A nivel más específico (Tabla 2), teniendo en cuenta las acciones que se llevan a cabo en cada uno de los factores que conforman la violencia escolar se constata que:

- En la violencia que ejerce el profesorado sobre el alumnado, hay coincidencia en las acciones violentas en la educación primaria y secundaria, con mayor presencia de bajar las notas al alumnado como castigo, tener manía a alguno de sus alumnos o castigar injustamente. Por el contrario, se muestra una menor presencia en acciones como ignorar, no escuchar, ridiculizar o insultar al alumnado. Las diferencias entre las medias $(\Delta M)$ oscilan entre $, 50 \mathrm{y}, 89$.

- En la violencia física entre el alumnado, las acciones de mayor presencia se centran en dar collejas o cachetes a compañeros bromeando, pegar o esconder pertenencias para fastidiar. Con menor asiduidad, acciones como robar objetos o dinero, agresiones físicas en cercanías del recinto escolar y esconder pertenencias o material del centro al profesorado para molestarlo deliberadamente. Destacar aquí, que la acción "los estudiantes pegan a compañeros/as dentro del recinto escolar" tiene mayor media en la educación primaria que secundaria. Las diferencias entre las medias $(\Delta M)$ oscilan entre, 18 y, 65 .

- En la violencia verbal entre el alumnado, las acciones se concentran en hablar mal unos de otros, extender rumores negativos, poner motes o insultar a compañeros/as. Por el contrario, las faltas de respeto o insultos al profesorado tienen una menor incidencia. Las diferencias entre las medias $(\Delta M)$ oscilan entre $, 32 \mathrm{y}, 65$, excepto en el ítem "El alumnado insulta a sus compañeros" que la media es ligeramente mayor en la etapa de primaria $(\Delta M=-, 05)$.

- La exclusión social, se concentra fundamentalmente en discriminación por buenos o malos resultados académicos, con una menor presencia de la discriminación por nacionalidad. Las diferencias entre las medias $(\Delta M)$ oscilan entre $, 06 \mathrm{y}, 47$. 
- En la disrupción en el aula, destacan acciones provocadas por el alumnado que no trabaja ni dejar trabajar al resto o dificulta las explicaciones del profesorado hablando durante la clase. Las diferencias entre las medias $(\Delta M)$ oscilan entre $, 20 \mathrm{y}, 56$.

- Finalmente, en la violencia ejercida a través de las tecnologías de la información y comunicación, se observa una mayor presencia de acciones encaminadas a enviar mensajes de ofensa, insulto o amenaza con el móvil o a través de redes sociales a compañeros/as, y grabar o publicar fotos para ofender o burlarse. En menor medida se presentan acciones como grabar o hacer fotos al profesorado para publicar en internet o burlarse de ellos. Las diferencias entre las medias $(\Delta M)$ oscilan entre $, 50 \mathrm{y}, 60$.

Tabla 2.

Medias y desviaciones típicas de los ítems de violencia escolar

\begin{tabular}{|c|c|c|c|c|c|c|}
\hline \multirow{2}{*}{\multicolumn{2}{|c|}{ ACCIONES VIOLENTAS }} & \multicolumn{2}{|c|}{ EP } & \multicolumn{2}{|c|}{ ESO } & \multirow{2}{*}{$\Delta M$} \\
\hline & & $M$ & DT & $M$ & $D T$ & \\
\hline \multirow{7}{*}{ VPA } & $\begin{array}{l}\text { 5. El profesorado } \\
\text { tiene manía a } \\
\text { algunos alumnos o } \\
\text { alumnas }\end{array}$ & 1.63 & .96 & 2.48 & 1.03 & .85 \\
\hline & $\begin{array}{l}\text { 9. El profesorado } \\
\text { ridiculiza al } \\
\text { alumnado }\end{array}$ & 1.24 & .67 & 1.74 & 1.02 & .50 \\
\hline & $\begin{array}{l}\text { 12. El profesorado } \\
\text { ignora a ciertos } \\
\text { alumnos o a ciertas } \\
\text { alumnas }\end{array}$ & 1.41 & .86 & 2.22 & 1.14 & .81 \\
\hline & $\begin{array}{l}\text { 15. El profesorado } \\
\text { castiga } \\
\text { injustamente }\end{array}$ & 1.56 & .84 & 2.45 & 1.20 & .89 \\
\hline & $\begin{array}{l}\text { 18. El profesorado } \\
\text { baja la nota a } \\
\text { algún o a alguna } \\
\text { estudiante como } \\
\text { castigo }\end{array}$ & 1.82 & .91 & 2.56 & 1.30 & .74 \\
\hline & $\begin{array}{l}\text { 23. El profesorado } \\
\text { insulta al } \\
\text { alumnado }\end{array}$ & 1.21 & .61 & 1.73 & 1.02 & .52 \\
\hline & $\begin{array}{l}\text { 27. El profesorado } \\
\text { no escucha a su } \\
\text { alumnado }\end{array}$ & 1.38 & .80 & 2.06 & 1.15 & .68 \\
\hline
\end{tabular}

2. Ciertos estudiantes roban objetos o dinero del $\begin{array}{lllll}1.51 & .82 & 1.79 & .97 & .28\end{array}$ centro educativo

16. Algunos estudiantes

VFA esconden pertenencias del profesorado o material del centro necesario en su trabajo, para molestarle deliberadamente

\begin{tabular}{lllllll}
\hline \multirow{2}{*}{ ACCIONES VIOLENTAS } & \multicolumn{3}{c}{ EP } & \multicolumn{2}{c}{ ESO } & \multirow{2}{*}{$\Delta M$} \\
\cline { 2 - 5 } & $M$ & $D T$ & $M$ & $D T$ & \\
\hline
\end{tabular}

19. Determinados

estudiantes dan

collejas o cachetes a

sus compañeros/as,

$\begin{array}{lllll}2.59 & 1.39 & 3.24 & 1.32 & .65\end{array}$

bromeando

21. Los estudiantes

pegan a

compañeros/as

dentro del recinto

escolar

VFA 25. Algunos

estudiantes

protagonizan

agresiones físicas en

$\begin{array}{lllll}1.53 & .71 & 1.87 & 1.10 & .34\end{array}$

las cercanías

del recinto escolar

26. Algunos

estudiantes

esconden

pertenencias de

$\begin{array}{lllll}2.67 & 1.08 & 2.06 & 1.16 & -.61\end{array}$

otros compañeros /

as, para fastidiar

1. El alumnado pone

motes molestos a sus

compañeros/as

$\begin{array}{lllll}2.02 & 1.01 & 2.59 & 1.16 & .57\end{array}$

3. Hay estudiantes

que extienden

rumores negativos

$\begin{array}{llll}2.45 & 1.17 & 2.78 & 1.17\end{array}$

.33

acerca de

compañeros/as

7. Los estudiantes

hablan mal unos de

otros

10. El alumnado falta al respeto a su profesorado en el

$\begin{array}{lllll}1.89 & .98 & 2.21 & 1.08 & .32\end{array}$

aula

14. Los estudiantes

insultan a

profesores/as

$\begin{array}{lllll}1.41 & .76 & 1.83 & 1.08 & .42\end{array}$

17. El alumnado

insulta a sus

$\begin{array}{lllll}2.48 & 1.18 & 2.43 & 1.11 & -.05\end{array}$

compañeros/as

\section{Algunos}

estudiantes son

discriminados por

ES sus compañeros/

as por sus buenos

resultados

académicos 


\begin{tabular}{|c|c|c|c|c|c|c|}
\hline \multirow{2}{*}{\multicolumn{2}{|c|}{ ACCIONES VIOLENTAS }} & \multicolumn{2}{|c|}{ EP } & \multicolumn{2}{|c|}{ ESO } & \multirow{2}{*}{$\Delta M$} \\
\hline & & $M$ & $D T$ & $M$ & $D T$ & \\
\hline \multirow[b]{2}{*}{ ES } & $\begin{array}{l}\text { 8. Determinados } \\
\text { estudiantes son } \\
\text { discriminados por } \\
\text { sus compañeros/as } \\
\text { por sus bajas notas }\end{array}$ & 1.86 & .97 & 1.92 & 1.11 & .06 \\
\hline & $\begin{array}{l}\text { 29. Algunos } \\
\text { estudiantes son } \\
\text { discriminados por } \\
\text { sus compañeros/ } \\
\text { as por su } \\
\text { nacionalidad }\end{array}$ & 1.53 & .86 & 1.95 & 1.14 & .42 \\
\hline \multirow{3}{*}{ DA } & $\begin{array}{l}\text { 11. El alumnado } \\
\text { dificulta las } \\
\text { explicaciones del } \\
\text { profesor/a con su } \\
\text { comportamiento } \\
\text { durante la clase }\end{array}$ & 2.54 & 1.07 & 3.01 & 1.14 & .47 \\
\hline & $\begin{array}{l}\text { 22. Hay alumnado } \\
\text { que ni trabaja ni } \\
\text { deja trabajar al } \\
\text { resto }\end{array}$ & 2.68 & 1.24 & 3.24 & 1.21 & .56 \\
\hline & $\begin{array}{l}\text { 30. El alumnado } \\
\text { dificulta las } \\
\text { explicaciones } \\
\text { del profesorado } \\
\text { hablando durante } \\
\text { la clase }\end{array}$ & 2.96 & 1.09 & 3.16 & 1.19 & .20 \\
\hline
\end{tabular}

\section{Algunos}

estudiantes graban

o hacen fotos a

compañeros/as

con el móvil, para

burlarse

\section{Ciertos}

estudiantes envían a compañeros / as mensajes con el móvil de ofensa, insulto o amenaza

VTIC 20. Hay estudiantes que graban o hacen fotos a profesores con el móvil, para burlarse de ellos

\section{Algunos} estudiantes envían mensajes a compañeros / a través de las redes sociales (Tuenti, Facebook,...) de ofensa, insulto o amenaza

\begin{tabular}{|c|c|c|c|c|c|}
\hline \multirow{2}{*}{ ACCIONES VIOLENTAS } & \multicolumn{2}{|c|}{ EP } & \multicolumn{2}{|c|}{ ESO } & \multirow{2}{*}{$\Delta M$} \\
\hline & $M$ & $D T$ & $M$ & $D T$ & \\
\hline $\begin{array}{l}\text { 28. Los estudiantes } \\
\text { publican en } \\
\text { Internet fotos o } \\
\text { vídeos ofensivos de } \\
\text { compañeros/as }\end{array}$ & 1.09 & .42 & 1.56 & .97 & .47 \\
\hline $\begin{array}{l}\text { 31. Los estudiantes } \\
\text { publican en } \\
\text { Internet fotos o } \\
\text { vídeos ofensivos de } \\
\text { profesores/as }\end{array}$ & 1.07 & .43 & 1.45 & .94 & .38 \\
\hline
\end{tabular}

Análisis inferencial de la violencia escolar en función del sexo, edad, etapa educativa y expediente académico

Los resultados de la prueba $t$ independiente realizados en la violencia escolar según el sexo (masculino o femenino) mostraron diferencias significativas en: la violencia que ejerce el profesorado sobre el alumnado $\left[t_{(1,1137)}=13,87 ; p<, 001\right]$, siendo mayor en hombres $\left(\eta^{2}=, 23\right)$; violencia verbal entre el alumnado $\left[t_{(1,1137)}=\right.$ $3,96 ; p<, 05]$ siendo mayor en mujeres $\left(\eta^{2}=, 12\right)$; exclusión social $\left[t_{(1,1137)}=3,89 ; p<, 05\right]$ siendo mayor en hombres $\left(\eta^{2}=, 31\right)$; y disrupción en el aula $\left[t_{(1,1137)}=4,06 ; p<, 05\right]$ siendo mayor en mujeres $\left(\eta^{2}=, 39\right)$. El tamaño de efecto es medio-bajo. No se presentan diferencias significativas en la violencia física del alumnado $\left[t_{1}\right.$ $\left.{ }_{1137)}=1,43 ; p>, 05\right]$ y la violencia a través de las tecnologías de la información y comunicación $\left[t_{(1,1137)}=1,75 ; p>, 05\right]$.

Si se tiene en consideración la edad (menos de 12 años, entre 12 y 14 años, más de 14 años) se observan diferencias significativas (prueba ANOVA) en todos los tipos de violencia escolar: violencia que ejerce el profesorado sobre el alumnado $\left[F_{(2,1136)}=\right.$ 98,76; $p<, 001]$, siendo mayor en el alumnado de edades entre 12 y 14 años $\left(\eta^{2}=, 92\right)$ o mayores de 14 años $\left(\eta^{2}=, 94\right)$, frente al alumnado con menos de 12 años; violencia física entre el alumnado $\left[F_{(2,1136)}=31,51 ; p<, 001\right]$, siendo mayor en el alumnado de edades entre 12 y 14 años $\left(\eta^{2}=, 43\right)$ o mayores de 14 años $\left(\eta^{2}=, 56\right)$, frente al alumnado con menos de 12 años; violencia verbal entre el alumnado $\left[F_{(2,1136)}=35,51 ; p<, 001\right]$, siendo mayor en el alumnado de edades entre 12 y 14 años $\left(\eta^{2}=, 51\right)$ o mayores de 14 años $\left(\eta^{2}=, 50\right)$, frente al alumnado con menos de 12 años; exclusión social $\left[F_{(2,1136)}=20,88 ; p<, 001\right]$, siendo mayor en el alumnado de edades entre 12 y 14 años $\left(\eta^{2}=, 41\right)$ o mayores de 14 años $\left(\eta^{2}=, 37\right)$, frente al alumnado con menos de 12 años; disrupción en el aula $\left[F_{(2,1136)}=51,41 ; p<, 001\right]$, siendo mayor en el alumnado de edades entre 12 y 14 años, frente al alumnado con menos de 12 años $\left(\eta^{2}=\right.$ ,65) o mayores de 14 años $\left(\eta^{2}=, 51\right)$; y violencia a través de las tecnologías de la información y comunicación $\left[F_{(2,1136)}=27,36 ; p<\right.$ ,001], siendo mayor en el alumnado de edades entre 12 y 14 años $\left(\eta^{2}=, 79\right)$ o mayores de 14 años $\left(\eta^{2}=, 96\right)$, frente al alumnado con menos de 12 años. El tamaño de efecto oscila entre medio y alto.

De igual forma, los resultados en la violencia escolar según la etapa educativa (primaria o secundaria) evidencian diferencias significativas en todas las variables: violencia que ejerce el profesorado sobre el alumnado $\left[t_{(1,1137)}=31,15 ; p<, 001\right]$, siendo mayor en educación secundaria $\left(\eta^{2}=, 98\right)$; violencia física entre el alumnado $\left[t_{(1,1137)}=39,39 ; p<, 001\right]$, siendo mayor en educación secundaria $\left(\eta^{2}=, 39\right)$; violencia verbal entre el alumnado $\left[t_{(1,1137)}=\right.$ $49,48 ; p<, 001]$, siendo mayor en educación secundaria $\left(\eta^{2}=, 44\right)$; exclusión social $\left[t_{(1,1137)}=37,08 ; p<, 001\right]$, siendo mayor en educación secundaria $\left(\eta^{2}=, 39\right)$; disrupción en el aula $\left[t_{(1,1137)}=48,89 ; p<\right.$ $, 001]$, siendo mayor en educación secundaria $\left(\eta^{2}=, 43\right)$; y violen- 
cia a través de las tecnologías de la información y comunicación $\left[t_{(1,1137)}=90,43 ; p<, 001\right]$, siendo mayor en educación secundaria $\left(\eta^{2}=, 92\right)$. El tamaño de efecto es medio-alto.

Finalmente, según el expediente del alumnado (ha aprobado siempre, ha suspendido alguna materia o ha repetido algún curso) también se manifiestan diferencias significativas (prueba ANOVA) en todos los tipos de violencia escolar: violencia que ejerce el profesorado sobre el alumnado $\left[F_{(2,1136)}=28,69 ; p<, 001\right]$, siendo mayor en el alumnado que suspende $\left(\eta^{2}=, 23\right)$ o repite $\left(\eta^{2}=51\right)$, frente al alumnado que aprueba, o del alumnado que repite frente al que suspende $\left(\eta^{2}=, 30\right)$; violencia física entre el alumnado $\left[F_{(2,1136)}=15,75 ; p<, 001\right]$, siendo mayor en el alumnado que suspende $\left(\eta^{2}=, 19\right)$ o repite $\left(\eta^{2}=, 38\right)$, frente al alumnado que aprueba, o del alumnado que repite frente al que suspende $\left(\eta^{2}=, 20\right)$; violencia verbal entre el alumnado $\left[F_{(2,1136)}=10,30 ; p<\right.$ ,001], siendo mayor en el alumnado que repite frente al alumnado que aprueba $\left(\eta^{2}=, 31\right)$; exclusión social $\left[F_{(2,1136)}=15,27 ; p<\right.$ ,001], siendo mayor en el alumnado que repite frente al alumnado que aprueba $\left(\eta^{2}=, 37\right)$; y violencia a través de las tecnologías de la información y comunicación $\left[F_{(2,1136)}=30,74 ; p<, 001\right]$, siendo mayor en el alumnado que repite frente al alumnado que aprueba $\left(\eta^{2}=, 51\right)$ o suspende $\left(\eta^{2}=, 37\right)$. El tamaño de efecto es medio-bajo. No se presentan diferencias significativas en la disrupción en el aula $\left[F_{(2,1136)}=1,83 ; p>, 05\right]$.

Análisis de regresión de las variables que constituyen la violencia escolar

El análisis de regresión realizado muestra que los modelos alcanzados son adecuados (La significación de F es menor de ,05 en todos ellos). Así pues, teniendo en cuenta las diferentes variables se evidencia que (Tabla 2):

- Las variables incluidas (significación t menor a ,05) en el modelo de la violencia ejercida por el profesorado hacia el alumnado explican el 48,4\% de la varianza, siendo las variables en orden de mayor a menor predicción: VFA, etapa educativa, VTIC, VVA y sexo. Quedan excluidas del modelo las variables ES, DA, edad y expediente académico (significación de $t$ mayor que ,05).

- En la violencia física por parte del alumnado (VFA), dicho modelo es explicado en un $62 \%$ por siete variables (significación $t$ menor a ,05). Las variables que se incluyen en el modelo ordenadas de mayor a menor presencia son: VVA, VTIC, VPA, Etapa educativa, DA, Edad y ES. Quedan excluidas las variables sexo y expediente académico (significación de $t$ mayor que ,05).

- En la violencia verbal entre el alumnado (VVA), dicho modelo es explicado en un $64,4 \%$ por siete variables (significación $t$ menor a ,05). Las variables que constituyen el modelo ordenadas de mayor a menor presencia son: VFA, DA, ES, VTIC, VPA, sexo y etapa educativa. Quedan excluidas del modelo las variables edad y expediente académico (significación de $\mathrm{t}$ mayor que ,05).

- En la exclusión social (ES), dicho modelo es explicado en un $44,7 \%$ por seis variables (significación t menor a ,05). Las variables que constituyen el modelo ordenadas de mayor a menor presencia son: VVA, VTIC, VFA, edad, expediente académico y sexo. No forman parte del modelo las variables VPA, DA y etapa educativa (significación de t mayor que ,05).

- En la disrupción en el aula (DA), dicho modelo es explicado en un $42,9 \%$ por seis variables (significación t menor a ,05). Las variables que constituyen el modelo ordenadas de mayor a menor presencia son: VVA, etapa educativa, VFA, edad, expediente académico y VTIC. Quedan excluidas del modelo las variables VPA, ES y sexo (significación de t mayor que ,05).

- En la violencia a través de las tecnologías de la información y comunicación (VTIC), dicho modelo es explicado en un $52,2 \%$ por seis variables (significación $t$ menor a ,05). Las variables que constituyen el modelo ordenadas de mayor a menor presencia son: VFA, ES, etapa educativa, VPA, VVA y DA. No forman parte del modelo las variables sexo, edad y expediente académico (significación de t mayor que ,05).

\section{Discusión y conclusiones}

Uno de los grandes retos que deben afrontar los centros educativos actuales es avanzar en las actitudes de respeto y no violencia, tratando de comprender los contextos en tensión y conflicto escolar. El punto de partida del estudio es el reconocimiento de las enormes carencias de las que adolece este ámbito de investigación, de enorme relevancia para un desarrollo armónico del sistema educativo. Pues bien, asumiendo la violencia escolar como aquella que describe actos considerados un obstáculo para el normal desarrollo de la actividad escolar (Ortega, 2012), se pretende conocer los tipos de violencia escolar en la educación primaria y secundaria, analizar la existencia de diferencias significativas de los factores que la conforman (VPA, VFA, VVA, ES, DA, VTIC) en función de las variables socio-escolares, y averiguar las variables de mayor capacidad predictiva que llevan a situaciones de violencia en centros educativos, se convierte en uno de los aspectos estratégicos para abordar el proceso de enseñanza-aprendizaje del alumnado.

En primer lugar, indicar que los datos alcanzados permiten confirmar, aunque con pequeñas matizaciones, la primera hipótesis de partida $\left(\mathrm{H}_{1}\right.$ Se espera obtener datos que confirmen la presencia de los mismos tipos de violencia en ambas etapas con mayor incremento de los mismos en la etapa de educación secundaria). Se constata una mayor presencia de violencia verbal, violencia que ejerce el profesorado y violencia física, en ambas etapas educativas. No obstante, dichas conductas violentas son más frecuentes en la etapa de educación secundaria. Estos datos justifican, parcialmente, investigaciones previas donde los tipos de violencia más habituales eran la violencia verbal y disrupción en el aula (Álvarez-García et al., 2014; Defensor del Pueblo-UNICEF, 2007; Díaz-Aguado, Martínez y Martín, 2010; Flores, 2009).

En este sentido, los resultados obtenidos focalizan mayor presencia de acciones violentas en los centros educativos encaminadas a bajar las notas al alumnado (como castigo), dar collejas o cachetes a compañeros bromeando, hablar mal o extender rumores negativos entre compañeros, discriminación por buenos o malos resultados académicos, no trabajar ni dejar trabajar a sus compañeros dificultado explicaciones o hablando durante las clases, y enviando a compañeros mensajes de ofensa, insulto o amenaza con el móvil o a través de redes sociales. De igual modo, todas las acciones de violencia que se producen en los centros educativos y se llevan a cabo en la etapa de educación primaria, se incrementan en la educación secundaria, excepto los hechos de pegar e insultar a compañeros dentro del recinto escolar, que disminuyen ligeramente en la etapa de educación secundaria.

En segundo lugar, nuestra segunda hipótesis $\left(\mathrm{H}_{2}\right.$ : Se espera que las variables socio-escolares -sexo, edad, etapa educativa y expediente académico-produzcan diferencias significativas en función de los factores que conforman la violencia escolar), se ha visto confirmada 
Tabla 3.

Análisis de regresión lineal de las variables criterio VPA, VFA, VVA, ES, DA, VTIC

\begin{tabular}{|c|c|c|c|c|c|c|c|c|c|c|}
\hline $\begin{array}{c}\text { Variable } \\
\text { Criterio } \\
\end{array}$ & & $R$ & & $R^{2}$ & $F$ & $\begin{array}{c}\text { Variables } \\
\text { Predictoras }\end{array}$ & $\boldsymbol{\beta}$ & $t$ & & $\begin{array}{c}\text { Varianza } \\
\text { Explicada } \\
\end{array}$ \\
\hline \multirow{5}{*}{ VPA } & \multirow{5}{*}{.696} & & \multirow{5}{*}{.484} & & \multirow{5}{*}{$\begin{array}{c}117.579 \\
(p=.000)\end{array}$} & VFA & .38 & 8.88 & $(p=.000)$ & $29.8 \%$ \\
\hline & & & & & & Etapa & .49 & 5.54 & $(p=.000)$ & $20.6 \%$ \\
\hline & & & & & & VTIC & .24 & 5.62 & $(p=.000)$ & $17.1 \%$ \\
\hline & & & & & & VVA & .15 & 3.48 & $(p=.479)$ & $12.4 \%$ \\
\hline & & & & & & Sexo & .27 & 4.98 & $(p=.012)$ & $10.9 \%$ \\
\hline $\begin{array}{l}\text { Variable } \\
\text { Criterio } \\
\end{array}$ & & $R$ & & $R^{2}$ & $F$ & $\begin{array}{c}\text { Variables } \\
\text { Predictoras }\end{array}$ & $\beta$ & $t$ & & $\begin{array}{c}\text { Varianza } \\
\text { Explicada }\end{array}$ \\
\hline \multirow{7}{*}{ VFA } & \multirow{7}{*}{.788} & & \multirow{7}{*}{.620} & & \multirow{7}{*}{$\begin{array}{c}204.80 \\
(p=.000)\end{array}$} & VVA & .32 & 11.59 & $(p=.000)$ & $33.7 \%$ \\
\hline & & & & & & VTIC & .24 & 8.59 & $(p=.000)$ & $22.1 \%$ \\
\hline & & & & & & VPA & .16 & 8.88 & $(p=.000)$ & $21.9 \%$ \\
\hline & & & & & & Etapa & -.85 & 6.22 & $(p=.000)$ & $19.8 \%$ \\
\hline & & & & & & DA & .23 & 6.48 & $(p=.000)$ & $15.5 \%$ \\
\hline & & & & & & Edad & .62 & 3.24 & $(p=.001)$ & $10.6 \%$ \\
\hline & & & & & & ES & .16 & 3.73 & $(p=.000)$ & $9.2 \%$ \\
\hline $\begin{array}{l}\text { Variable } \\
\text { Criterio }\end{array}$ & & $R$ & & $R^{2}$ & $F$ & $\begin{array}{l}\text { Variables } \\
\text { Predictoras }\end{array}$ & $\beta$ & $t$ & & $\begin{array}{c}\text { Varianza } \\
\text { Explicada }\end{array}$ \\
\hline \multirow{7}{*}{ VVA } & \multirow{7}{*}{.803} & & \multirow{7}{*}{.644} & & \multirow{7}{*}{$\begin{array}{l}227.287 \\
(p=.000)\end{array}$} & VFA & .33 & 11.59 & $(p=.000)$ & $31.6 \%$ \\
\hline & & & & & & DA & .48 & 13.69 & $(p=.000)$ & $29.8 \%$ \\
\hline & & & & & & ES & .42 & 9.84 & $(p=.000)$ & $22.5 \%$ \\
\hline & & & & & & VTIC & .12 & 4.29 & $(p=.000)$ & $10.9 \%$ \\
\hline & & & & & & VPA & .07 & 3.48 & $(p=.001)$ & $8.6 \%$ \\
\hline & & & & & & Sexo & -.72 & 4.20 & $(p=.000)$ & $7.7 \%$ \\
\hline & & & & & & Etapa & -.71 & 2.33 & $(p=.001)$ & $7.3 \%$ \\
\hline $\begin{array}{l}\text { Variable } \\
\text { Criterio } \\
\end{array}$ & & $R$ & & $R^{2}$ & $F$ & $\begin{array}{c}\text { Variables } \\
\text { Predictoras }\end{array}$ & $\boldsymbol{\beta}$ & $t$ & & $\begin{array}{c}\text { Varianza } \\
\text { Explicada } \\
\end{array}$ \\
\hline \multirow{6}{*}{ ES } & \multirow{6}{*}{.669} & & \multirow{6}{*}{.447} & & \multirow{6}{*}{$\begin{array}{l}101.386 \\
(p=.000)\end{array}$} & VVA & .18 & 9.84 & $(p=.000)$ & $35.1 \%$ \\
\hline & & & & & & VTIC & .17 & 9.12 & $(p=.000)$ & $28.2 \%$ \\
\hline & & & & & & VFA & .07 & 3.73 & $(p=.000)$ & $13.3 \%$ \\
\hline & & & & & & Edad & -.33 & 2.60 & $(p=.009)$ & $10.3 \%$ \\
\hline & & & & & & Exp. Académ. & .16 & 2.24 & $(p=.025)$ & $5.6 \%$ \\
\hline & & & & & & Género & .27 & 2.35 & $(p=.019)$ & $5.4 \%$ \\
\hline $\begin{array}{l}\text { Variable } \\
\text { Criterio }\end{array}$ & & $R$ & & $R^{2}$ & $F$ & $\begin{array}{l}\text { Variables } \\
\text { Predictoras }\end{array}$ & $\beta$ & $t$ & & $\begin{array}{l}\text { Varianza } \\
\text { Explicada }\end{array}$ \\
\hline \multirow{6}{*}{ DA } & \multirow{6}{*}{.655} & & \multirow{6}{*}{.429} & & \multirow{6}{*}{$\begin{array}{c}94.112 \\
(p=.000)\end{array}$} & VVA & .29 & 13.69 & $(p=.000)$ & $47.8 \%$ \\
\hline & & & & & & Etapa & .54 & 6.54 & $(p=.000)$ & $25.5 \%$ \\
\hline & & & & & & VFA & .15 & 6.48 & $(p=.000)$ & $23.3 \%$ \\
\hline & & & & & & Edad & -.64 & 4.21 & $(p=.000)$ & $16.9 \%$ \\
\hline & & & & & & Exp. Académ. & -.42 & 4.86 & $(p=.000)$ & $12.3 \%$ \\
\hline & & & & & & VTIC & -.08 & 3.63 & $(p=.000)$ & $11.8 \%$ \\
\hline $\begin{array}{l}\text { Variable } \\
\text { Criterio } \\
\end{array}$ & & $R$ & & $R^{2}$ & $F$ & $\begin{array}{c}\text { Variables } \\
\text { Predictoras } \\
\end{array}$ & $\boldsymbol{\beta}$ & $t$ & & $\begin{array}{c}\text { Varianza } \\
\text { Explicada } \\
\end{array}$ \\
\hline \multirow{6}{*}{ VTIC } & \multirow{6}{*}{.722} & & & & & VFA & .25 & 8.59 & $(p=.000)$ & $27.8 \%$ \\
\hline & & & & & & ES & .39 & 9.12 & $(p=.000)$ & $24.4 \%$ \\
\hline & & & 522 & & 136.749 & Etapa & .65 & 5.51 & $(p=.000)$ & $19.8 \%$ \\
\hline & & & & & $(p=.000)$ & VPA & .11 & 5.62 & $(p=.000)$ & $15.9 \%$ \\
\hline & & & & & & VVA & .12 & 4.29 & $(p=.000)$ & $14.7 \%$ \\
\hline & & & & & & DA & -.13 & 3.63 & $(p=.000)$ & $9.8 \%$ \\
\hline
\end{tabular}

Nota: VPA: Violencia del Profesor hacia el Alumnado; VFA: Violencia Física por parte del Alumnado; VVA: Violencia Verbal del Alumnado; ES: Exclusión Social; DA: Disrupción en el Aula; VTIC: Violencia a través de las Tecnologías de la Información y la Comunicación. 
en gran medida, ya que los resultados ratifican diferencias significativas en el sexo (excepto en la violencia física del alumnado y la violencia a través de las tecnologías de la información y comunicación), etapa educativa, edad y expediente académico (excepto la disrupción en el aula).

Así pues, en referencia a la violencia escolar según el sexo, los datos dan cuenta que el sexo masculino tiene una mayor presencia en la violencia del profesorado y la exclusión social, mientras que el femenino lo hace en la violencia verbal y disrupción en el aula. Estos resultados están en la línea del estudio realizado por Núñez, Álvarez-García, Rodríguez, González-Castro, Álvarez y González-Pienda (2010) al sostener que el sexo masculino percibe un mayor nivel de violencia del profesorado hacia el alumnado, o el llevado a cabo por el Defensor del Pueblo-UNICEF (2007) que evidencia una mayor violencia verbal en el sexo femenino. Asimismo, investigaciones realizadas por Archer y Coyne (2005) y Card, Stucky, Sawalani y Little (2008) evidencian que los chicos utilizaban en mayor medida la agresión directa (agresiones físicas y verbales), mientras que las chicas utilizaban más la agresión indirecta (exclusión social y rechazo). No obstante, contradice el estudio elaborado por Ararteko-IDEA (2006) que considera una mayor participación de los hombres en conductas disruptivas.

En la misma línea, teniendo en consideración la edad, se revela que a menor edad su implicación en situaciones de violencia será más baja en todos sus tipos. Sin embargo, como excepción debemos señalar que la disrupción en el aula se da preferentemente el alumnado cuya edad oscila entre los 12 y 14 años. Son numerosos los estudios (Álvarez-García et al., 2011; Calvete, Orue, Estévez, Villardón y Padilla, 2010; Fernández, Álvarez, Ceña y Álvarez-García, 2010; Garaigordobil y Oñederra, 2009; Síndic de Greuges, 2007) que señalan una variación del tipo de violencia escolar en función de la edad, con mayor presencia de violencia física a menor edad.

Respecto a la etapa educativa, los datos sostienen que la educación secundaria obligatoria presenta un mayor índice de incidencia en todos los tipos de violencia escolar. Así pues, estudios realizados en la etapa de secundaria muestran diferencias en el tipo de violencia, indicando que la violencia física se concentra en el primer y segundo curso de secundaria (Fernández et al., 2010), que una mayor incidencia de la violencia a través de las TIC se manifiesta en segundo curso de secundaria (Calvete et al., 2010; Ortega, Calmaestra y Mor 2008) o que el alumnado del primer ciclo de secundaria dice ser víctima de exclusión social, violencia verbal directa y violencia física directa (Defensor del Pueblo-UNICEF, 2007).

Si se tiene en cuenta el expediente educativo del alumnado, se constata una mayor presencia de todos los tipos de violencia en el alumnado repetidor (excepto en la disrupción en el aula). Estos resultados son coincidentes con otras investigaciones que afirman la existencia de un rendimiento más bajo en el alumnado inmerso en situaciones de violencia escolar (Álvarez-García, Rodríguez, Gonzalez-Castro, Núñez y Álvarez, 2010; Cerezo, 2001).

En cuanto a la capacidad de predicción, los datos alcanzados confirman parcialmente nuestra tercera hipótesis $\left(\mathrm{H}_{3}: \mathrm{La} V P A\right.$, $V F A, V V A, E S, D A, V T I C$, sexo, edad, etapa educativa y expediente académico serán predictores de violencia en los centros educativos). En consecuencia, la violencia escolar en las etapas de educación primaria y secundaria se puede predecir en mayor medida por las variables: violencia verbal, violencia física, etapa educativa y violencia a través de las tecnologías de la información y comunicación. Estos resultados confirman la investigación realizada por Nieto (2019) quien sostiene que la violencia escolar se predice en mayor medida por la violencia física directa por parte del alumnado, violencia a través de las nuevas tecnologías de la información y comunicación, y violencia verbal entre el alumnado; o parcialmente en el trabajo de Domínguez, Álvarez y Vázquez (2017) al afirmar que se puede predecir fundamentalmente por la violencia entre el alumnado y la violencia ejercida por el alumnado hacia el profesorado.

Finalmente, indicar que este trabajo guarda enorme interés para la comunidad educativa, pues pretende conocer la percepción del alumnado de educación primaria y secundaria sobre la violencia en los centros de enseñanza y analizar cuáles son los tipos de violencia escolar de mayor relevancia y si estos difieren en función de determinadas variables socio-escolares. Dichos hallazgos suponen elementos de primer orden que sientan las bases de la actividad profesional docente para una mejor convivencia escolar. Estas revelaciones deberían ser consideradas desde los responsables de las instituciones educativas, así como por parte del profesorado, si se tiene en cuenta que serán los encargados de formar a las próximas generaciones en ambientes libres de violencia. Respecto a las limitaciones del estudio, sería conveniente señalar algunas cuestiones a tener en cuenta a la hora de interpretar los resultados. Es muy difícil aislar cada uno de los factores que conforman el constructo violencia escolar, por ello no se está en disposición de asegurar si son estos factores y no otros los que minimizan las situaciones de violencia en los centros educativos. Tampoco se sabe en qué medida los adolescentes se implican, profunda o superficialmente, en la cumplimentación de los cuestionarios y sinceridad de sus respuestas.

Sería conveniente que futuras investigaciones incluyan información, tanto cualitativa como cuantitativa, que proporcione testimonios directos o indirectos sobre la violencia escolar, con aportaciones interrelacionadas de familias, profesorado y alumnado. De hecho, sería importante mitigar el impacto negativo de los diversos tipos de violencia en el desempeño escolar, incorporando fundamentalmente tratamientos preventivos. Así pues, los tipos e intensidad de violencia escolar identificados en este estudio, pueden servir como referencias de focalización que permitan identificar los centros más vulnerables y favorecer la convivencia en los mismos.

\section{Referencias}

Albaladejo-Blázquez, N., Ferrer-Cascales, R., Reig-Ferrer, A. y Fernández-Pascual, M.D. (2013) ¿Existe violencia escolar en educación infantil y primaria? Una propuesta para su evaluación y gestión? Anales de Psicología, 29(3), 1060-1069. https:/ / doi. org/10.6018/analesps.29.3.158431

Álvarez-García, D., Álvarez, L., Núñez, J.C., González-Pienda, J.A., González-Castro, P. y Rodríguez, C. (2008). Estudio del nivel de violencia escolar en siete centros asturianos de Educación Secundaria. Aula Abierta, 36(1, 2), 89-96.

Álvarez-García, D., Núñez, J.C. y Dobarro, A. (2013). Cuestionarios para evaluar la violencia escolar en Educación Primaria y en Educación Secundaria: CUVE3-EP y CUVE3-ESO. Apuntes de Psicología, 31, 191-202.

Álvarez-García, D., Dobarro, A., Álvarez, L., Núñez, J.C. y Rodríguez, C. (2014). La violencia escolar en los centros de educación secundaria de Asturias desde la perspectiva del alumnado. Educación XXI, 17(2), 337-360. https:/ / doi.org/10.5944/educxx1.17.2.11494

Álvarez-García, D., Núñez, J. C., Rodríguez, C., Álvarez, L. y Dobarro, A. (2011). Propiedades psicométricas del Cuestionario de Violencia Escolar-Revisado (CUVE-R). Revista de Psicodidáctica, 16, 59-83. 
Álvarez-García, D., Rodríguez, C., González-Castro, P., Núñez, J.C. y Álvarez, L. (2010). La formación de los futuros docentes frente a la violencia escolar. Revista de Psicodidáctica, 15(1), 35-56.

Ararteko-IDEA (2006). Convivencia y conflictos en los centros educativos. Vitoria-Gasteiz: Ararteko.

Archer, J. y Coyne, S. M. (2005). An integrated review of indirect, relational, and social aggression. Personality and Social Psychology Review, 9(3), 212-230. https://doi.org/10.1207/ s15327957pspr0903_2

Ayala-Carrillo, M.R. (2015). Violencia escolar: Un problema complejo. Ra Ximhai, 11(4), 493-509.

Bisquerra, R. (2014). Metodología de la Investigación Educativa. Madrid: La Muralla.

Blaya, C. y Debarbieux, É. (2011). La violencia en los colegios de enseñanza primaria: ¿cómo están los alumnos franceses? MAGIS, Revista Internacional de Investigación en Educación, 4(8), 339-356.

Calderón, N.G., Vera, J.A. y Llano, O.D. (2018). La violencia escolar desde la perspectiva de los estudiantes de una escuela secundaria de Sonora, México. Paideia, 62, 107-125.

Calmaestra, J., Escorial, A., García, P., Del Moral, C., Perazzo, C. y Ubrich, T. (2016). Yo a eso no juego. Madrid: Save the Children.

Calvete, E., Orue, I., Estévez, A., Villardón, L. y Padilla, P. (2010). Cyberbullying in adolescents: Modalities and aggressors' profile. Computers in Human Behavior, 26(5), 1128-1135. https:/ / doi.org/10.1016/j.chb.2010.03.017.

Card, N. A., Stucky, B. D., Sawalani, G. M. y Little, T. D. (2008). Direct and indirect aggression during childhood and adolescence: A meta-analytic review of gender differences, intercorrelations, and relations to maladjustment. Child Development, 79(5), 1185-1229. https://doi.org/10.1111/j.1467-8624.2008.01184.x

Ceballos, E., Correa-Rodríguez, N., Correa-Piñero, A., Rodríguez-Hernández, J., Rodríguez-Ruiz, B. y Vega, A. (2012). La voz del alumnado en el conflicto escolar. Revista de Educación, 359, 554-579.

Cerezo, R. (2001). La Violencia en las Aulas. Madrid: Pirámide.

Cerezo, F. (2009). Analyzing Bullying in Spanish School. International Journal of Psychology and Psychological Therapy, 9(3), 383-394.

Cerezo-Ramirez, F. (2017). Bullying a traves de las TIC. Boletín Científico Sapiens Research, 2(2), 24-29.

Coarite, E., y G. Carrillo (2014). Consideraciones sobre la violencia escolar y los conflictos de estudiantes, Revista Integra Educativa, 7(2), 175-186.

Cohen, J. (1990). Things I Have Learned (So Far). American Psychologist, 45(12), 1304-1312.

Conde, S. y Ávila, J.A. (2019). El maltrato escolar en centros de educación primaria en la provincia de Huelva (España). Psicodebate, 18(1), 51-71. http:/ / dx.doi.org/10.18682/pd.v18i1.743.

Debarbieux, E. y Blaya, C. (2010). Sociología y violencia escolar: un enfoque contextual. En R. Ortega (Coord.), Agresividad injustificada, bullying y violencia escolar (pp. 355-383). Madrid: Alianza Editorial.

Defensor del Pueblo-UNICEF (2007). Violencia escolar: el maltrato entre iguales en la educación secundaria obligatoria. 1999-2006. Madrid: Publicaciones de la Oficina del Defensor del Pueblo.

Díaz-Aguado, M.J., Martínez, R. y Martín, J. (2010). Estudio estatal sobre la convivencia escolar en la Educación Secundaria Obligatoria. Madrid: Ministerio de Educación, Observatorio Estatal de la Convivencia.

Domínguez, J., Álvarez, E. y Vázquez, E. (2017). Dimensiones predictivas del constructo violencia escolar en la educación se- cundaria obligatoria. Revista de Investigación Educativa, RIE, 35(2), 337-351. https://doi.org/10.6018/rie.35.2.259471.

Domínguez-Alonso, J., López-Castedo, A. y Nieto-Campos, B. (2019). Violencia escolar: diferencias de género en estudiantes de secundaria. Revista Complutense De Educación, 30(4), 10311044. https://doi.org/10.5209/rced.59997

Falla, D., Alós, F.J., Moriana, J.A. y Ortega, R. (2012). La violencia entre estudiantes según el profesorado en los Centros de Educación Especial de Córdoba. Aula Abierta, 40(1), 3-14.

Fernández, G., Álvarez, L., Ceña, F.J. y Álvarez-García, D. (2010). La conflictividad escolar en Asturias. Propuestas de intervención. Gijón: Centro del Profesorado y de Recursos de Avilés.

Flores, K. (2009). El derecho a una vida escolar sin violencia. La Paz: Asociación Voces Vitales.

Garaigordobil, M. y Oñederra, J. A. (2009). Un análisis del acoso escolar desde una perspectiva de género y grupo. Ansiedad y Estrés, 15, 193-205.

Garaigordobil, M., Martínez, V. y Machimbarrena, J.M. (2017). Intervención en el bullying y cy-berbullying: Evaluación del caso Martín. Revista de Psicología Clínica con Niños y Adolescentes, 4(1), 25-32.

García-Fernández, C.M., Romera-Félix, E.M. y Ortega-Ruiz, R. (2017). Cyberbullying en Educación Primaria: Factores explicativos relacionados con los distintos roles de implicación. Psychology, Society and Education, 9(2), 251-262. https://doi. org/10.25115/psye.v9i2.697

Giménez, A.M., Arnaiz, P., Cerezo, F. y Prodocimo, E. (2018). Percepción de docentes y estudiantes sobre el ciberacoso. Estrategias de intervención y afrontamiento en Educación Primaria y Secundaria. Comunicar, 56(XXVI), 29-38. https://doi. org/10.3916/C56-2018-03

González-Medina, M.A. y Treviño-Villarreal, D.C. (2019). Violencia escolar en bachillerato: algunas estrategias para su prevención desde diferentes perspectivas. Revista Interuniversitaria, 31(1), 123. https: / / doi.org/10.14201/teri.19616

Gutiérrez, N. (2019). Aproximación al estudio de los tipos de violencia escolar percibidos por el alumnado universitario. Revista de Psicología y Ciencias del Comportamiento de la Unidad Académica de Ciencias Jurídicas y Sociales, 10(1), 103-114. https:/ / doi. org/10.29059/rpcc.20190602-84.

IBM Corp. (2012). SPSS Statistics for Windows, Version 21.0. Armonk, NY: IBM Corp.

Instituto Vasco de Evaluación e Investigación Educativa (2012). El maltrato entre iguales en educación primaria y ESO. Bilbao: ISEI.IVEI.

Jares, X.R. (2006). Pedagogía de la convivencia. Barcelona: Graó.

Jiménez, T. y Lehalle, H. (2012). La violencia escolar entre iguales en alumnos populares y rechazados. Psychosocial Intervention, 21(1), 77-89. https:/ / doi.org/10.5093/in2012v21n1a5.

Juvonen, J. y Graham, S. (2014). Bullying in schools: The power of bullies and the plight of victims. Annual Review of Psychology, 65, 159-185. https://doi.org/10.1146/annurev-psych-010213-115030.

Kim, B.E., Gilman, A.B., Hill, K.G. y Hawkins, J.D. (2016). Examining protective factors against violence among high-risk youth: Findings from the Seattle Social Development Project. Journal of Criminal Justice, 45, 19-25.

Kuppens, S., Grietens, H., Onghena, P., Michiels, D. y Subramanian, S.V. (2008). Individual and classroom variables associated with relational aggression in elementary-school aged children: A multilevel analysis. Journal of School Psychology, 46, 639-660.

León-Moreno, C., Martínez-Ferrer, B., Musitu-Ochoa, G. y David Moreno-Ruiz, D. (2019). Victimisation and School Violen- 
ce. The Role of the Motivation of Revenge, Avoidance, and Benevolence in Adolescents. Revista de Psicodidáctica (English ed.), 24(2), 88-94. https://doi.org/10.1016/j.psicod.2018.12.001.

Liébana, J.A., Deu del Olmo, M.I. y Real, S. (2015). Valoración del conocimiento sobre el maltrato infantil del profesorado ceutí. Revista Española de Orientación y Psicopedagogía, 26(1), 100-114.

Loeber, R., Capaldi, D.M. y Costello, E. (2013). Gender and the development of aggression, disruptive behavior, and delinquency from childhood to early adulthood. In P. Tolan \& B. Leventhal, Disruptive behavior disorders (pp. 137-160). New York: Springer.

López, J. y Fernández, I. (2000). Coexistence in Educational Centers: Spain's Situation. España: Ministerio de Educación y Ciencia.

López, M. (2012). El bullying en la escuela: un asunto de grandes y chicos. Bogotá (Colombia): Ediciones Gamma S.A.

Martínez-Baena, A. y Faus-Bosca, J. (2018). Acoso escolar y Educación Física: una revisión sistemática. Retos, 34, 338-345.

Medina, J.A. y Reverte, M.J. (2019). Violencia escolar, rasgos de prevalencia en la victimización individual y grupal en la Educación Obligatoria en España. REXE. Revista de Estudios y Experiencias en Educación, 18(37), 97-110. https://doi.org/10.21703/ rexe. 20191837 medina9.

Merma, G., Peiró, S. y Gavilán, D. (2013). Perspectivas sobre Educación en Valores en Tiempos de Crisis. Barataria. Revista Castellano-Manchega de Ciencias Sociales, 15, 151-160.

Nieto, B. (2019). Violencia escolar e inteligencia emocional en adolescentes gallegos. Tesis Doctoral (Escola Internacional de Doutoramento). Universidade de Vigo: Pontevedra.

Núñez, J.C., Álvarez-García, D., Rodríguez, C., González-Castro, P., Álvarez, L. y González-Pienda, J. A. (2010). Diferencias de género en el nivel de violencia escolar percibido por los estudiantes de Educación Secundaria. En J.J. Gázquez y M.C. Pérez (Eds.) Investigación en convivencia escolar. Variables relacionadas (pp. 181-182). Granada: Grupo Editorial Universitario.

Olweus, D. (2006). Una revisión general. En Á. Serrano (Ed.), Acoso y Violencia en la Escuela (pp. 79-103). Madrid: Editorial Ariel.

Ortega, R. (2012). Normativa escolar: análisis a los manuales de convivencia escolar de los establecimientos educacionales municipales de la Comuna de Santiago. (Trabajo de pregrado). Universidad de Chile, Chile. Recuperado de: http://www.tesis.uchile.cl/handle/2250/11404
Ortega, R., Calmaestra, J. y Mora, J. (2008). Cyberbullying. International Journal of Psychology and Psychological Therapy, 8(2), 183-192.

Pacheco-Salazar, B. (2018). Violencia escolar: la perspectiva de estudiantes y docentes. Revista Electrónica de Investigación Educativa, 20(1), 112-121. https: / / doi.org/10.24320/redie.2018.20.1.1523

Pérez, A.J. (2015). Influencia de la actividad física sobre conductas violentas en adolescentes de centros educativos públicos de Granada. Tesis Doctoral. Universidad de Granada: Granada.

Real Academia Española de la Lengua (2019). Diccionario de la Lengua Española (Actualización 2019). Madrid: Espasa Libros, S.L.U.

Rivera, J.P., Reynoso, T.M. y Vilchis, R.M. (2018). Efectos de un programa de ciberconvivencia en la prevención del cyberbullying. Psychology, Society E Education, 10(2), 239-250. https:/ / doi. org/10.25115/psye.v10i1.1953

Salazar, J.A.R. y González, S. (2019). De la micro-violencia al clima escolar: claves de comprensión desde el discurso de profesores. Psicoperspectivas, 18(1), 25. http://doi.org/10.5027/psicoperspectivas-vol18-issue1-fulltext-1559

Sánchez, I.M., Vallejo, E.I.G. y Roig, M.R. (2019). El acoso escolar en educación secundaria: prevalencia y abordaje a través de un estudio de caso. Social y ciencias sociales. Comunitania: Revista Internacional de Trabajo, 17, 71-91.

Sapién-Zúñiga, L., Ledezma-Rivas, P. y Ramos-Trevizo, J. (2019). Percepción de la violencia escolar en el último ciclo de educación primaria. RECIE. Revista Electrónica Científica de Investigación Educativa, 4(2), 1349-1360.

Síndic de Greuges (2007). La escuela: espacio de convivencia y conflictos. Alicante: Síndic de Greuges de la Comunitat Valenciana.

UNESCO (2019). Behind the numbers: Ending school violence and bullying. France: United Nations Educational, Scientific and Cultural Organization.

Weimer, W.R. y Moreira, E.C. (2014). Violencia e bullying: manifestaçoes e consequencias nas aulas de Educacao Fisica escolar. Revista Brasileira de Ciências do Esporte, 36(1), 257-274. 
